\title{
Side-information-aided Non-coherent Beam Alignment Design for Millimeter Wave Systems
}

\author{
Yi Zhang, Kartik Patel, Sanjay Shakkottai, and Robert W. Heath Jr. \\ The University of Texas at Austin, Austin, Texas, USA \\ \{yi.zhang.cn,kartikpatel,rheath\}@utexas.edu,shakkott@austin.utexas.edu
}

\begin{abstract}
Designing efficient and robust beam alignment strategies for millimeter wave (mmWave) systems is important for overcoming training overheads and practical hardware impairments. In this work, we leverage side information in the form of prior knowledge of the angular support of the propagation channel (direction information) to design a compressive sensing (CS) based beam alignment algorithm. Existing CS based channel estimation approaches assume perfect phase information of the measurements, which is not the case with the low-cost off-the-shelf mmWave phased arrays. Instead, we develop a two-stage algorithm where we use the magnitude of measurements (aka non-coherent measurements); using phase retrieval (PR) followed by sparse recovery, we estimate the channel gain across various (quantized) spatial angles. To validate the proposed algorithm, we develop a fully reconfigurable mmWave testbed with custom-made 2-bit phased arrays. We perform a careful calibration to the phased arrays, thus enabling generations of precise desired beam patterns. Our implementation and real experiments validate both the proposed algorithm and calibration process by demonstrating consistency between the experimental results and the theoretical analysis.
\end{abstract}

\section{CCS CONCEPTS}

- Computer systems organization $\rightarrow$ Reconfigurable computing; • Hardware $\rightarrow$ Beamforming; Digital signal processing; - Networks $\rightarrow$ Mobile ad hoc networks.

\section{KEYWORDS}

$60 \mathrm{GHz}$, Beam Alignment, Non-Coherent, Compressive Phase Retrieval, Side Information, Prototyping, Calibration

\section{ACM Reference Format:}

Yi Zhang, Kartik Patel, Sanjay Shakkottai, and Robert W. Heath Jr.. 2019. Sideinformation-aided Non-coherent Beam Alignment Design for Millimeter Wave Systems. In Mobihoc '19: The Twentieth ACM International Symposium on Mobile Ad Hoc Networking and Computing, July 2-5, 2019, Catania, Italy. ACM, New York, NY, USA, 10 pages. https://doi.org/10.1145/3323679.3326532

Permission to make digital or hard copies of all or part of this work for personal or classroom use is granted without fee provided that copies are not made or distributed for profit or commercial advantage and that copies bear this notice and the full citation on the first page. Copyrights for components of this work owned by others than the author(s) must be honored. Abstracting with credit is permitted. To copy otherwise, or republish, to post on servers or to redistribute to lists, requires prior specific permission and/or a fee. Request permissions from permissions@acm.org.

Mobihoc '19, July 2-5, 2019, Catania, Italy

(c) 2019 Copyright held by the owner/author(s). Publication rights licensed to ACM ACM ISBN 978-1-4503-6764-6/19/07 ..\$15.00

https://doi.org/10.1145/3323679.3326532

\section{INTRODUCTION}

MmWave communication now enables flexible transmission with its large available spectrum resources [23]. The small wavelengths at mmWave frequencies and the recent developments in the semiconductor industry are able to compact large antennas to form electronically-steerable phased arrays with small physical sizes. This provides more flexibility to configure antenna elements to get favorable received signal power and desired radiation patterns [30]. Configuring these phased arrays, however, have significant challenges. First, the use of highly directional beams results in large beam training overhead [18]. Second, it is challenging to develop silicon-based antenna systems that can generate perfect beam patterns without any irregularities [25] and fulfill the other performance requirements posed by the existing compressive channel estimation algorithms [30]. Therefore, designing efficient beam training algorithms that are compatible with low-cost hardware is crucial for enabling mmWave communication in practice.

To reduce the beam training overhead, one promising solution is to leverage side information from available sensors to infer the channel prior to performing any training [12]. This helps to avoid probing the potentially ineffective beams. Among various sensor data, position information can be obtained by positioning systems like commercial GPS, or out-of-band communication systems [18, 27]. In the line-of-sight (LOS) scenarios, the location information of the transmitter $(\mathrm{Tx})$ and receiver $(\mathrm{Rx})$ can be directly used to infer the angle of departure (AoD) and angle of arrival (AoA) of the LOS path. In the non-line-of-sight (NLOS) scenarios, a mechanism similar to the sector level sweep (SLS) in IEEE 802.11ad can be used to infer the AoD and AoA of a dominant path [17]. In this work, we will use side information to help to estimate the direction of the dominant channel path, hence reducing the beam training overhead.

Several mmWave beamforming and channel estimation algorithms have been developed without considering the imperfection of practical devices or hardware impairments $[4,31]$. The real performance of these algorithms is thus rarely examined by practical experiments. For low-cost phased arrays, large phase noise and irregularities of beam patterns do not satisfy the assumption of perfect coherence across all training slots (packets), that is typically required by current compressive designs. This was experimentally confirmed by [21]. It was reported in [14] that an accumulated random and unknown phase corrupts the phase information of the measurements across packets. Due to the unstable oscillators of low-cost devices, there exists an unknown carrier frequency offset between the local oscillators at the transceivers, which is dynamically changing $[20,21,25]$. These unstable oscillators result in an accumulated phase noise which destroys the phase information of the measurement. In particular, for low-bandwidth mmWave 
communication, e.g. IoT application, the impact of phase noise will be even stronger. Given these hardware limitations, we propose to only leverage the magnitude of measurements, which is termed as non-coherent measurements, to design a beam alignment algorithm.

Motivated by these observations, in this work, we leverage prior knowledge of the dominant channel path direction to design a noncoherent mmWave beam alignment algorithm that is compatible with the low-cost off-the-shelf mmWave phased arrays. To experimentally validate the proposed algorithm, we develop a MATLABbased software defined radio (SDR) platform for fast prototyping with fully reconfigurable phased arrays which are carefully calibrated. Our algorithms and experiments focus on a narrowband mmWave setting ( $5 \mathrm{MHz}$ bandwidth in our experiments) that is especially well-suited to emerging low-cost IoT applications. The major contributions of this work are summarized as follows:

1. Side-information-aided non-coherent beam alignment algorithm design. we develop a two-stage compressive phase retrieval (CPR) algorithm to estimate the channel only with the magnitude of training measurements. In particular, we incorporate side information in the form of prior knowledge of the angle information of the dominant path, to reduce the beam training overhead. With our side-information-aided non-coherent beam alignment algorithm, a better estimation of the direction of the dominant path is achieved in comparison with the traditional beam sweeping strategy. To the best of our knowledge, this is the first work that demonstrates the feasibility of the CPR technique in estimating the mmWave channel with side information and non-coherent measurements.

2. Reconfigurable mmWave testbed and experimental validation. To examine the proposed side-information-aided noncoherent beam alignment algorithm, we develop a low-cost mmWave testbed using SiBEAM $60 \mathrm{GHz}$ phased arrays and USRPs. We use MATLAB to develop a phased array control interface and to perform baseband signal processing. With this testbed, we experimentally validate the proposed algorithm. Our experiments demonstrate consistency between the experimental results and the theoretical analysis of the proposed algorithm. In addition, the related code is publicly accessible in [35], which can provide a starting point for researchers interested in developing mmWave testbeds or prototyping advanced mmWave algorithms.

3. MmWave phased array calibration. As is well known, compressive channel estimation and beamforming relies on knowing the exact radiation patterns. Therefore, a small irregularity in beam pattern generation can result in unpredictable and undesired errors in compressive channel estimation, further worsening the link performance through beam misalignment. Indeed, prior mmWave prototyping studies have specifically reported the unexpected irregularity of the beam patterns generated by the off-the-shelf phased arrays [24, 25]. Therefore, phased array calibration is necessary to guarantee that the desired beam patterns are applied. In this work, we develop a one-time phase array calibration. Our experiments show that the performed calibration improved the received signal-to-noise ratio (SNR) by $4 \mathrm{~dB}$. To the best of our knowledge, this is the first mmWave prototype with phased array calibration, which makes the beam pattern generation flexibly reconfigurable and accurate.

\section{SYSTEM MODEL}

We consider a mmWave system consisting of a single transmitter equipped with a uniform linear array (ULA) of $N_{\mathrm{Tx}}$ antennas and a single receiver equipped with a ULA of $N_{\mathrm{Rx}}$ antennas. We let $s$ be the transmitted training symbol such that $\mathbb{E}\left[|s|^{2}\right]=1$ and let a $N_{\mathrm{Rx}} \times N_{\mathrm{Tx}}$ matrix $\mathrm{H}$ represent the channel between the Tx and $\mathrm{Rx}$. For the $n$-th beam training slot ( $n$-th measurement), the Tx uses a $N_{\mathrm{Tx}} \times 1 \mathrm{RF}$ precoder $\mathrm{f}_{n}$ and the Rx applies a $N_{\mathrm{Rx}} \times 1 \mathrm{RF}$ combiner $\mathbf{w}_{n}$. Due to the low-cost fabrications of the off-the-shelf phased arrays and the unstable oscillators between transceivers, there exists stochastic phase noise across different training packets, which we denote as $\epsilon_{n}$. By adopting a frequency-flat channel model, the received signal $\tilde{y}_{n}$ can be expressed as

$$
\tilde{y}_{n}=\mathbf{w}_{n}^{*} \mathbf{H f}_{n} e^{j \epsilon_{n}} s+\mathbf{w}_{n}^{*} \mathbf{n},
$$

where $\mathbf{n}$ is additive noise which follows $C \mathcal{N}\left(0, \sigma^{2} \mathbf{I}_{N_{\mathrm{Rx}}}\right)$. Since this accumulated phase noise $e^{j \epsilon_{n}}$ corrupts the phase information of the measurement, we only use the magnitude of $\tilde{y}_{n}$ to estimate the channel, hence transforming (1) into

$$
y_{n}=\left|\tilde{y}_{n}\right|=\left|\mathbf{w}_{n}^{*} \mathbf{H f}_{n} s+\mathbf{w}_{n}^{*} \mathbf{n} e^{-j \epsilon_{n}}\right| .
$$

Since multiplying $\mathbf{n}$ by $e^{j \epsilon_{n}}$ does not change its distribution, we use $\mathbf{n}$ to represent $\mathbf{n} e^{-j \epsilon_{n}}$ in the following. Therefore, the non-coherent measurement observed in the $n$-the training slot is given by

$$
y_{n}=\left|\mathbf{w}_{n}^{*} \mathbf{H f}_{n} s+\mathbf{w}_{n}^{*} \mathbf{n}\right| .
$$

In this work, we adopt a geometric channel model with $L$ independent propagation paths between the Tx and Rx. We assume that all scattering happens in azimuth and only 1-D beamforming is implemented in the studied system. Then, $l$-th path is parameterized by $\left\{g_{\ell}, \phi_{\ell}, \theta_{\ell}\right\}$, where $g_{\ell}$ is the complex channel gain (including path loss) of the $\ell$-th path, $\phi_{\ell} \in\left[-90^{\circ}, 90^{\circ}\right]$ and $\theta_{\ell} \in\left[-90^{\circ}, 90^{\circ}\right]$ are the physical azimuth AoD and AoA of the $\ell$-th path. In particular, $\phi_{\ell}$ and $\theta_{\ell}$ are the angles with respect to the broadside of ULA. Under this model, the $N_{\mathrm{Rx}} \times N_{\mathrm{Tx}}$ channel matrix $\mathrm{H}$ can be written as

$$
\mathbf{H}=\sqrt{N_{\mathrm{Tx}} N_{\mathrm{Rx}}} \sum_{\ell=1}^{L} g_{\ell} \mathbf{a}_{\mathrm{Rx}}\left(\theta_{\ell}\right) \mathbf{a}_{\mathrm{Tx}}^{H}\left(\phi_{\ell}\right),
$$

where $\mathbf{a}_{\mathrm{Tx}}\left(\phi_{\ell}\right)$ and $\mathrm{a}_{\mathrm{Rx}}\left(\theta_{\ell}\right)$ represent the antenna array response vector at the Tx and Rx side, respectively. The expression of $\mathbf{a}_{\mathrm{Tx}}\left(\phi_{\ell}\right)$ is given as

$\mathbf{a}_{\mathrm{Tx}}\left(\phi_{\ell}\right)=\frac{1}{\sqrt{N_{\mathrm{Tx}}}}\left[1, e^{-j 2 \pi \frac{d}{\lambda} \sin \left(\phi_{\ell}\right)}, \ldots, e^{-j\left(N_{\mathrm{Tx}}-1\right) 2 \pi \frac{d}{\lambda} \sin \left(\phi_{\ell}\right)}\right]^{T}$,

where $d$ is the spacing distance between two neighboring antenna elements and $\lambda$ is the wavelength of the signal. The array response vector of the $\mathrm{Rx}$ is defined in a similar manner.

\section{PROBLEM FORMULATION}

In this section, we first recap the sparse representation of the generic mmWave channel estimation problem with non-coherent measurements. Then we introduce our way of incorporating side information into the formulated problem. 


\subsection{Sparse representation of non-coherent mmWave channel estimation}

Based on the signal model provided in Section 2, we now derive a sparse formulation of the non-coherent channel estimation problem. Suppose that the Tx transmits the energy-normalized training symbol $\left\{s_{p}\right\}_{p=1}^{M_{\mathrm{Tx}}}$ with $M_{\mathrm{Tx}}$ different precoders $\left\{\mathbf{f}_{p}\right\}_{p=1}^{M_{\mathrm{Tx}}}$, and for each precoder, the Rx applies $M_{\mathrm{Rx}}$ distinct combiners $\left\{\mathbf{w}_{q}\right\}_{q=1}^{M_{\mathrm{Rx}}}$ to receive the signal, then the measurement can be rewritten as

$$
y_{q, p}=\left|\mathbf{w}_{q}^{*} \mathbf{H f}_{p} s_{p}+\mathbf{w}_{q}^{*} \mathbf{n}\right| .
$$

We denote $\mathbf{F}=\left[\mathbf{f}_{1}, \ldots, \mathbf{f}_{M_{\mathrm{TX}}}\right]$ and $\mathbf{W}=\left[\mathbf{w}_{1}, \ldots, \mathbf{w}_{M_{\mathrm{Rx}}}\right]$. We let the AoD and AoA be uniformly quantized by $G_{\mathrm{Tx}}$ and $G_{\mathrm{Rx}}$ levels, respectively. Then we let $A_{\mathrm{D}}$ represent an $N_{\mathrm{Tx}} N_{\mathrm{Rx}} \times G_{\mathrm{Tx}} G_{\mathrm{Rx}}$ dictionary matrix consisting of $G_{\mathrm{Tx}} G_{\mathrm{Rx}}$ column vectors of the form $\left(\mathrm{a}_{\mathrm{Tx}}^{*}\left(\bar{\phi}_{u}\right) \otimes \mathrm{a}_{\mathrm{Rx}}\left(\bar{\theta}_{v}\right)\right)$, where $\otimes$ is the Kronecker product operator, $\bar{\phi}_{u}$ and $\bar{\theta}_{v}$ denote the $u$-th and $v$-th uniform quantization level of AoD and AoA, respectively. Based on these, according to [4], we can vectorize the $M_{\mathrm{Tx}} M_{\mathrm{Rx}}$ training measurements in (6) as

$$
\mathbf{y}_{\mathbf{v}}=\left|\left(\mathbf{F}^{T} \otimes \mathbf{W}^{*}\right) \mathbf{A}_{\mathrm{D}} \mathbf{z}+\mathbf{n}_{\mathrm{V}}\right|,
$$

where $y_{q, p}$ is the $\left[(p-1) M_{\mathrm{Rx}}+q\right]$-th element of $\mathbf{y}_{\mathrm{V}}, \mathbf{n}_{\mathrm{V}}$ is the vectorized noise, and $\mathrm{z}$ represents the complex-valued path gain of the corresponding quantized spatial angles. The formulation in (7) demonstrates a sparse representation of the mmWave channel since $\mathrm{z}$ has $L$ non-zero elements [4] and $L \ll G_{\mathrm{Tx}} G_{\mathrm{Rx}}$, which further leads to a CPR problem.

\subsection{Application of side information}

In this subsection, we discuss the form of side information and apply it to revising the non-coherent measurements in (7). We assume that with existing positioning services or out-of-band communication modules, the AoD and AoA of the dominant channel path are known to lie in searching regions $S_{\phi} \triangleq[\hat{\phi}, \hat{\phi}+\Delta \phi]$ and $S_{\theta} \triangleq[\hat{\theta}, \hat{\theta}+\Delta \theta]$, respectively. Here $S_{\phi}$ and $S_{\theta}$ are subsets of $\left[-90^{\circ}, 90^{\circ}\right]$, and $\Delta \phi$ and $\Delta \theta$ are termed as searching range of the AoD and AoA.

We now denote $\tilde{\mathbf{z}}$ as a vector which is composed of the elements, from $\mathrm{z}$, that correspond to the searching regions of the AoD and AoA. Then we denote $\tilde{\mathbf{z}}^{\mathrm{c}}$ as a vector consisting of the remaining elements of z. Similarly, we can denote $\tilde{A}_{D}$ and $\tilde{A}_{D}^{c}$ as the sub dictionary matrices corresponding to $\tilde{\mathbf{z}}$ and $\tilde{\mathbf{z}}^{\mathrm{c}}$, respectively. Then (7) is written as

$$
\mathbf{y}_{\mathbf{v}}=\left|\left(\mathbf{F}^{T} \otimes \mathbf{W}^{*}\right) \tilde{\mathbf{A}}_{\mathrm{D}} \tilde{\mathbf{z}}+\left(\mathbf{F}^{T} \otimes \mathbf{W}^{*}\right) \tilde{\mathbf{A}}_{\mathrm{D}}^{\mathrm{c}} \tilde{\mathbf{z}}^{\mathrm{c}}+\mathbf{n}_{\mathbf{V}}\right| .
$$

Under the assumption that the dominant path is much stronger than the other paths, we can treat the term $\left(\mathbf{F}^{T} \otimes \mathbf{W}^{*}\right) \tilde{A}_{\mathrm{D}}^{\mathrm{c}} \tilde{\mathbf{z}}^{\mathrm{c}}$ as part of noise, which yields

$$
\mathbf{y}_{\mathbf{V}}=\left|\left(\mathbf{F}^{T} \otimes \mathbf{W}^{*}\right) \tilde{\mathbf{A}}_{\mathrm{D}} \tilde{\mathbf{z}}+\tilde{\mathbf{n}}_{\mathbf{V}}\right|,
$$

where $\tilde{\mathbf{n}}_{\mathbf{V}}=\left(\mathbf{F}^{T} \otimes \mathbf{W}^{*}\right) \tilde{\mathbf{A}}_{\mathrm{D}}^{\mathrm{c}} \tilde{\mathbf{z}}^{\mathrm{c}}+\mathbf{n}_{\mathbf{V}}$. Actually, focusing on $\tilde{\mathbf{z}}$ rather than $\mathrm{z}$ means that we only consider the dominant path of the channel. This is reasonable since we are performing analog beamforming and only one path will be used for data transmission. It is worth pointing out that the incorporation of side information, i.e. from (8) to (9), is closely related to the performance of the following proposed non-coherent algorithm because this helps to reduce the training overheads as the dimensionality of $\tilde{\mathbf{z}}$ is not greater than $\mathbf{z}$. We denote the size of $\tilde{\mathbf{z}}$ as $N \times 1$.

\section{NON-COHERENT BEAM ALIGNMENT ALGORITHM}

In this section, a two-stage algorithm is proposed to estimate the sparse vector $\tilde{\mathbf{z}}$ (the complex-valued path gain of the quantized angles in the searching regions) in (9) with the non-coherent measurements $\mathbf{y}_{\mathrm{v}}$. For notation simplicity, we define a sensing matrix $\Psi \triangleq\left(\mathrm{F}^{T} \otimes \mathbf{W}^{*}\right) \tilde{\mathrm{A}}_{\mathrm{D}}$ for (9). The proposed algorithm is inspired by a state-of-the-art CPR algorithm provided by [5] and [15], in which there are no restrictions for their sensing matrices. In the context of mmWave systems, the sensing matrix $\Psi$ is constrained by the features of phased arrays, such as low-resolution phase shifters, a limited number of RF chains, and etc. Accordingly, we will design a particular sensing matrix $\Psi$ which fits our formulated non-coherent estimation problem and meanwhile maintains the benefit of the state-of-the-art CPR algorithm.

\subsection{CPR based channel estimation}

In this subsection, we will present a two-stage algorithm to recover $\tilde{z}$. Suppose that the sensing matrix $\Psi$ can be decomposed into a product of a PR matrix of size $M_{\mathrm{Rx}} M_{\mathrm{Tx}} \times M_{\mathrm{CS}}$, denoted by $\Psi_{\mathrm{PR}}$, and a CS matrix of size $M_{\mathrm{CS}} \times N$, denoted by $\Psi_{\mathrm{CS}}$, which is expressed as

$$
\Psi=\Psi_{\mathrm{PR}} \Psi_{\mathrm{CS}} .
$$

In particular, we require that $M_{\mathrm{Rx}} M_{\mathrm{Tx}}>M_{\mathrm{CS}}$ and $M_{\mathrm{CS}}<N$, namely that the sensing matrix $\Psi$ is decomposed into a product of two low-rank matrices. When $\Psi$ is not directly decomposable, a low-rank approximation will be performed to it, which will be detailed in the next subsection. We further denote the noise, with respect to $\mathbf{y}_{\mathrm{v}}$, introduced by the low-rank approximation as $\mathbf{r}$. Then the measurement vector $\mathbf{y}_{\mathrm{v}}$ in (9) can be written as

$$
\mathbf{y}=\left|\Psi_{\mathrm{PR}} \Psi_{\mathrm{CS}} \tilde{\mathbf{z}}+\tilde{\mathbf{n}}_{\mathrm{V}}+\mathbf{r}\right|^{2},
$$

where $\mathrm{y} \triangleq \mathrm{y}_{\mathrm{v}}^{2}$. To avoid calculating the squared magnitude with the added noise terms, we remodel $\mathrm{y}$ as

$$
\mathbf{y}=\left|\Psi_{\mathrm{PR}} \Psi_{\mathrm{CS}} \tilde{\mathbf{z}}\right|^{2}+\mathbf{e},
$$

where $\mathbf{e}$ is an overall noise term bounded as $\|\mathbf{e}\|_{2} \leq \varepsilon$. This error model was proposed by [7] and it is suitable for our case. This is because our measurements are corrupted by multiple random noise sources consisting of the signal from the paths out of the searching region, i.e. $\Psi \tilde{\mathbf{z}}^{\mathrm{c}}$, the residual from the low-rank approximation, and the thermal noise $\mathbf{n}_{\mathbf{V}}$.

We now introduce an intermediate vector $\mathbf{y}_{C S} \triangleq \Psi_{C S} \tilde{z}$, of size $M_{\mathrm{CS}} \times 1$. Then the CPR estimation problem in (12) can be decomposed into two concatenated problems given by

$$
\begin{aligned}
& \mathbf{y}=\left|\Psi_{\mathrm{PR}} \mathbf{y}_{C S}\right|^{2}+\mathbf{e}, \\
& \mathbf{y}_{\mathrm{CS}}=\Psi_{\mathrm{CS}} \tilde{\mathbf{z}} .
\end{aligned}
$$

First of all, (13a) represents a PR problem without requiring $\mathbf{y}_{C S}$ to have a sparse property. In addition, we have $M_{\mathrm{Rx}} M_{\mathrm{Tx}}>M_{\mathrm{CS}}$, which fits the requirement of solving a classic PR problem, that the number of measurements should be larger than the problem's 
dimensionality. The rule of thumb is that the number of measurements is more than 4 times as the problem's dimensionality [2]. Thus, we can retrieve $y_{C S}$ by solving (13a) given $M_{\mathrm{Rx}} M_{\mathrm{Tx}}>M_{\mathrm{CS}}$. Secondly, it can be seen that (13b) is a traditional CS problem with $M_{\mathrm{CS}}<N$, namely that the sparse vector $\tilde{\mathbf{z}}$, of size $N \times 1$, is to be recovered via a smaller number of measurements $y_{\mathrm{CS}}$, of size $M_{\mathrm{CS}} \times 1$. As a result, the decomposition in (10) reduces the original CPR problem in (12) into a PR problem with a smaller dimensionality of $M_{\mathrm{CS}}$ and a traditional CS problem. The newly generated two problems in (13) are solved sequentially in two stages illustrated in the following.

Stage 1 Phase retrieval: In this work, we use the PhaseLift approach proposed in $[7,8]$ to solve the PR problem formulated in (13a). By lifting the target sparse vector $\mathbf{y}_{C S}$ as $\mathbf{Y}_{C S}=\mathbf{y}_{C S} \mathbf{y}_{C S}^{*}$, (13a) can be transformed as a regularized convex optimization problem given as [7]

$$
\begin{aligned}
& \min _{\mathrm{Y}_{\mathrm{CS}}} \frac{1}{2}\left\|\mathrm{y}-\operatorname{diag}\left(\Psi_{\mathrm{PR}} \mathrm{Y}_{\mathrm{CS}} \Psi_{\mathrm{PR}}^{*}\right)\right\|_{2}^{2}+\eta \operatorname{Tr}\left(\mathrm{Y}_{\mathrm{CS}}\right) \\
& \text { s.t. } \mathrm{Y}_{\mathrm{CS}} \geq 0,
\end{aligned}
$$

where $\eta$ is a positive scalar that is chosen depending on the level of noise. After solving the problem (14), we use the largest eigenvector of $\mathrm{Y}_{\mathrm{CS}}$ as an estimate of $\mathbf{y}_{\mathrm{CS}}$.

Stage 2 Sparse estimation: With the estimated $y_{C S}$ from stage 1 , the next step is to recover the sparse vector $\tilde{\mathbf{z}}$ in (13b), corresponding to the complex-valued path gains along the quantized angles in the searching regions. Classic CS algorithms can be applied to estimate $\tilde{\mathbf{z}}$, such as the OMP method [28] or the EMBGAMP method [32].

\subsection{Generation and low-rank decomposition of sensing matrix}

We now discuss the generation of $\Psi_{\mathrm{PR}}$ and $\Psi_{\mathrm{CS}}$. Overall, we propose to use a sensing matrix generated by overlapped directional beams (instead of a random sensing matrix that is more typical in literature). The motivations of this are: (1) With side information, the Tx and Rx can probe directional beams within the known searching region, which provides a higher receiver SNR than the traditional CS-based channel estimation where random beam patterns are used. (2) Directional beams are easier to calibrate and thus the regularity of their radiation patterns is easier to guarantee, in comparison with randomly generated beams. Accordingly, for low-cost devices, even with a fixed codebook of directional beams, better beam alignment can be performed. (3) It also turns out numerically that the proposed two-stage algorithm does not work well with a random sensing matrix.

Taking the generation of the precoder matrix $\mathbf{F}$ as an example, the idea is to generate $M_{\mathrm{Tx}}$ directional beams that fully covers the searching region of AoD. To this end, we uniformly partition $S_{\phi}$ into $M_{\mathrm{Tx}}$ sub-regions and then the $M_{\mathrm{Tx}}$ physical pointing directions are set to the centers of the $M_{\mathrm{Tx}}$ sub-regions. Then $\mathrm{F}$ is constructed by $M_{\mathrm{Tx}}$ array response vector corresponding to these $M_{\mathrm{Tx}}$ directions. This is further quantized according to the resolution of the phase shifters. In the following, we use $\mathbf{F}$ to represent the precoder after quantization. To fully cover the searching region, a certain overlap between neighboring beams is required. Since the beamwidth of the directional beams generated by a ULA with $N_{\mathrm{Tx}}$ elements can be roughly estimated as $\frac{102}{N_{\mathrm{Tx}}}$ (in degrees), we set that $\frac{102 M_{\mathrm{Tx}}}{N_{\mathrm{Tx}}}>$ $\Delta \phi$ to guarantee the performance of the proposed algorithm. The quantized combiner $\mathbf{W}$ is calculated in a similar manner.

Given the precoder and combiner, we can have the sensing matrix $\Psi=\left(\mathbf{F}^{T} \otimes \mathbf{W}^{*}\right) \tilde{A}_{\mathrm{D}}$. To obtain the PR matrix $\Psi$ and the CS matrix $\Psi$, we perform the SVD to $\Psi$, which yields

$$
\Psi=\mathrm{U} \Sigma \mathrm{V}^{*} \text {. }
$$

We then keep the top $M_{\mathrm{CS}}$ left and right singular vectors and the top $M_{\mathrm{CS}}$ singular values of $\Psi$, namely we compute a low-rank approximation of $\Psi$ via SVD given as

$$
\Psi \approx \mathrm{U}_{M_{\mathrm{CS}}} \Sigma_{M_{\mathrm{CS}}} \mathrm{V}_{M_{\mathrm{CS}}}^{*}=\mathrm{U}_{M_{\mathrm{CS}}} \sqrt{\Sigma_{M_{\mathrm{CS}}}} \sqrt{\Sigma_{M_{\mathrm{CS}}}} \mathrm{V}_{M_{\mathrm{CS}}}^{*},
$$

which yields

$$
\begin{aligned}
& \Psi_{\mathrm{PR}}=\mathrm{U}_{M_{\mathrm{CS}}} \sqrt{\Sigma_{M_{\mathrm{CS}}}}, \\
& \Psi_{\mathrm{CS}}=\sqrt{\Sigma_{M_{\mathrm{CS}}}} \mathbf{V}_{M_{\mathrm{CS}}}^{*} .
\end{aligned}
$$

Choosing $M_{\mathrm{CS}}$ is crucial because a larger value for $M_{\mathrm{CS}}$ would make a better approximation of the sensing matrix $\Psi$ but results in worse performance in recovering $\mathbf{y}_{\mathrm{CS}}$ as the problem's dimensionality of the PR problem in (13a) is $M_{\mathrm{CS}}$. The choice of $M_{\mathrm{CS}}$ is expected to be as small as possible subject to obtaining a useful approximation. To evaluate the performance of the low-rank approximation of $\Psi$, we define $\gamma$ as the ratio of the sum of the singular values being kept to the sum of all the singular values, which is expressed as $\gamma=\frac{\operatorname{Tr}\left(\Sigma_{M_{\mathrm{CS}}}\right)}{\operatorname{Tr}(\Sigma)}$. Intuitively, $\gamma$ is a metric that indicates how much principal component of the target matrix is captured by SVD. In our application, we choose $\gamma$ within $[0.8,0.9]$. The proposed side-information-aided non-coherent estimation algorithm is summarized in Algorithm 1.

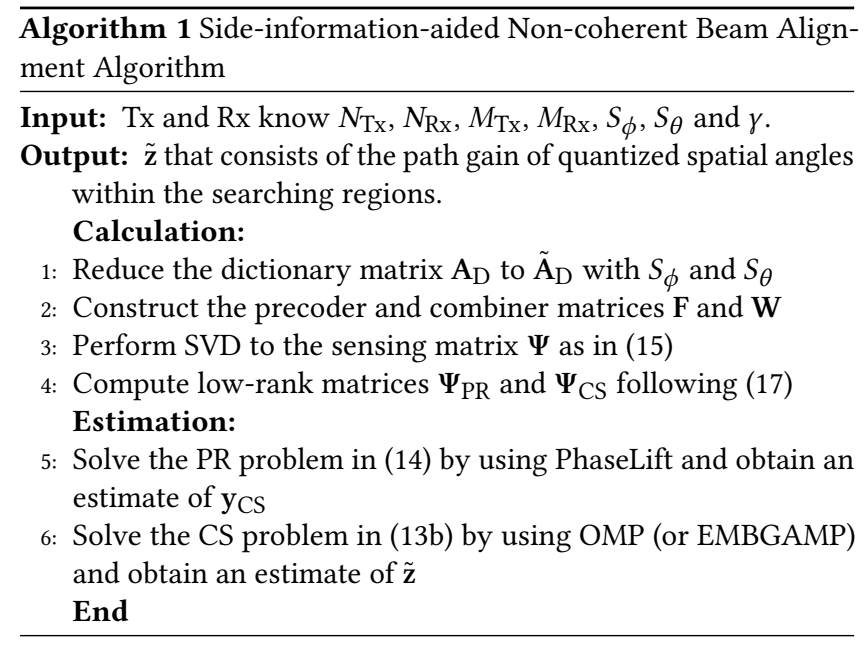

\section{EXPERIMENTAL SETUP}

In this section, we present our mmWave testbed. Firstly, the hardware components are summarized. Secondly, the hardware setup 


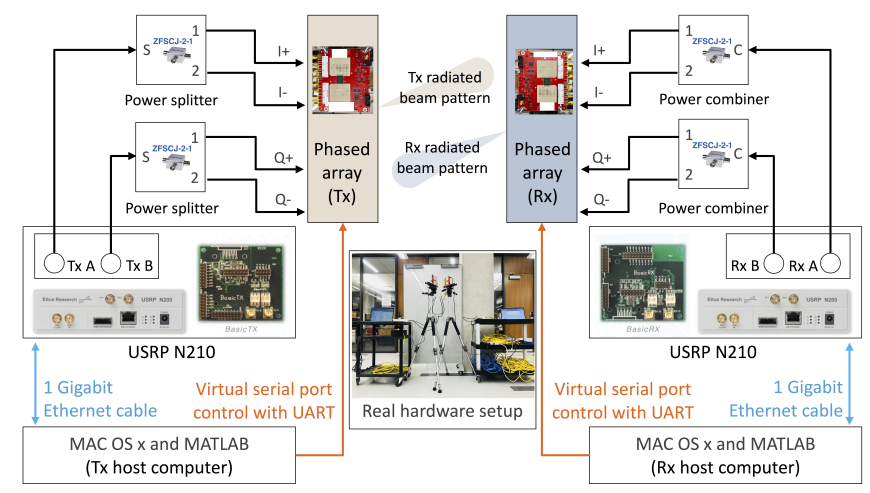

Figure 1: Block diagram of the mmWave testbed

and a software-controlled system are introduced. Finally, the procedure of data collection is illustrated.

\subsection{Key hardware components}

Two USRP N210 each is used at both the Tx and the Rx sides to connect one phased array and one host computer. In addition, we use SiBEAM Sil6342 $60 \mathrm{GHz}$ phased arrays, donated by Google Inc. They are 12-element 2-bit phased arrays operating at 60.48/62.64 $\mathrm{GHz}$ with the antenna spacing $3.055 \mathrm{~mm}$. This setup supports narrowband mmWave communications, with a bandwidth of $5 \mathrm{MHz}$.

\subsection{Hardware setup}

A block diagram with the hardware connection is given in Fig. 1. Taking the connection at the Tx side as an example: (1) The Tx USRP generates the baseband signal, which is fed into the Tx phased array via 2 way $-180^{\circ}$ power splitters. (2) The phased arrays are mounted onto camera tripods, which allows azimuthal rotation. This makes the arrays stand at a height of 1.7 meters above the ground. (3) The phased arrays are connected to the corresponding host computers via USB cables and are further controlled via virtual serial ports. The universal asynchronous receiver-transmitter (UART) protocol is applied to read the status of phased arrays and more importantly to reconfigure them (antenna switching on/off, phase shifters' states/codebook and RF gain). A similar assembly is done at the $\mathrm{Rx}$ side.

\subsection{Software setup}

There are two software modules. One is a general purpose processor (GPP) based SDR platform. We use MATLAB to connect and configure USRPs for data exchange. This module includes baseband signal processing as well. The other module is the control interface for the phased arrays. This interface is developed using serial port programming (C language), which is called by MATLAB. Since a GPP based SDR and the phased array control is used together during the experiment, it is important to ensure a successful reconfiguration of the phased arrays before taking the measurements. Thereby, the control interface waits for a certain period ( $3 \mathrm{~ms}$ in our case) after sending each command for reconfiguration.

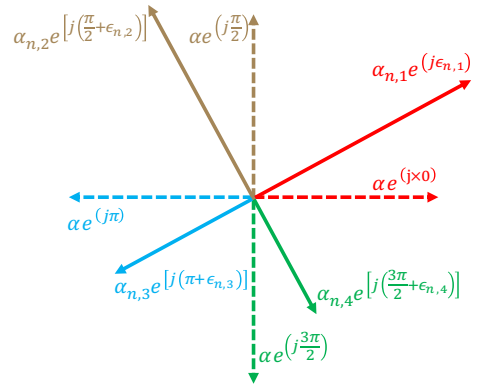

Figure 2: Ideal and real radiation properties of each nominal phase state of $n$-th antenna. The solid arrows represent the real magnitudes and phases of the nominal phase states while the dashed arrows are the ideal ones.

\subsection{Procedure of data collection}

To begin the data collection process, the Tx program is started by configuring the Tx USRP. Then the Tx phased array is reconfigured, which involves setting the on/off state of each antenna and the phase state of each phase shifter, i.e. applying the desired codebook. After having successfully reconfigured the Tx phased array, we feed the training data to the Tx USRP and start the transmission. Afterward, the Rx program is started and similar operations are performed. It is important to point out that the reconfiguration of the $\mathrm{Tx}(\mathrm{Rx})$ phased array is always prior to starting signal transmission (reception). This is to ensure that the collected measurements are correctly transmitted and received under the desired configurations of the phased arrays. In our experiments, one measurement refers to the averaged result of one received packet consisting of 10000 samples under QPSK modulation with $5 \mathrm{Ms} / \mathrm{s}$ sampling rate.

\section{PHASED ARRAY CALIBRATION}

In this section, we briefly describe a one-time calibration process that is performed to our mmWave testbed. Calibrating a phased array refers to determining the magnitude and phase behaviors of each antenna. Only with carefully calibrated phased arrays, we can produce desired beam patterns accurately. The SiBeam's phased array provides each antenna element with four phase states $0^{\circ}, 90^{\circ}$, $180^{\circ}$ and $270^{\circ}$. The real phases, however, are not precisely the nominal values due to the low-cost fabrication of its PCB. Specifically, Fig. 2 depicts the potential imperfection of a 2-bit phased array. Therein, four nominal phase states are shown by the dashed arrows and the real phase states with undesired magnitudes and shifted phases are represented by the solid arrows.

\subsection{Phase match based calibration}

In this part, we describe our phase match based calibration (PMBC). We refer to Fig. 2 for notation. We denote the magnitude of radiation pattern when only the $r$-th phase state of the $n$-th antenna element is activated as $\alpha_{n, r}$ and its phase as $e^{j(r-1) \frac{\pi}{2}+\epsilon_{n, r}}$, where $\epsilon_{n, r}$ is the phase error with respect to the nominal phase $(r-1) \frac{\pi}{2}$. Note that $\alpha_{n, r}$ and $e^{j(r-1) \frac{\pi}{2}+\epsilon_{n, r}}$ are relative magnitude and phase with respect to those of a reference phase state (RPS) of a reference antenna (RA). Without loss of generality, we set the 1-st antenna as 


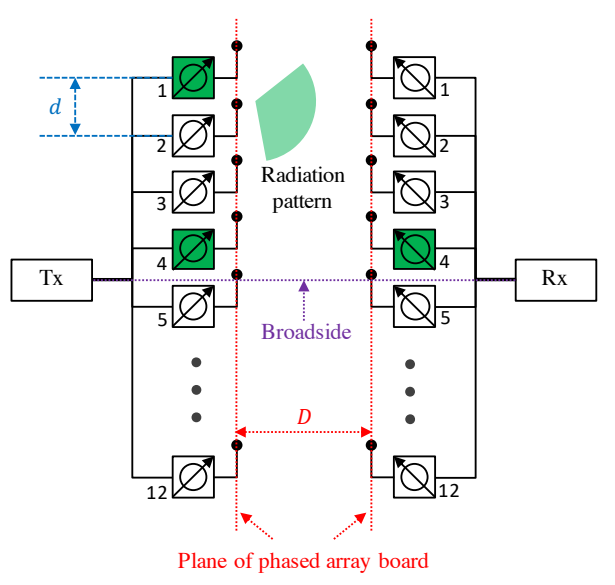

Figure 3: Schematic of the mechanical alignment, where $d$ is the antenna element spacing and $D$ is the distance between two phased array boards. This schematic is not proportional as $D$ is much larger than $d$. In addition, this schematic also serves as an example of calibrating the 4th $T x$ antenna, where the color green indicates that the antenna is activated.

the RA and its 1-st phase state as the RPS. For simplicity, we further set that $\alpha_{1,1}=1$.

A sequential calibration process of our proposed $\mathrm{PMBC}$ is summarized as follows: (1) The mechanical alignment must be first conducted to make the planes of Tx and Rx phased array board perfectly face to each other, as shown in Fig. 3. (2) The magnitude calibration can be easily accomplished by switching an antenna on one at a time and measuring its antenna gain. (3) The phase calibration is performed by activating two antenna elements: one antenna is serving as a reference antenna (RA) and the other one is the antenna to be calibrated (ATC), for which an example is given in Fig. 3. By enumerating all the nominal phase states of the ATC, four measurements are collected. Given the far-field model and that the $\mathrm{RA}$ is set to the 1-st antenna with the reference phase state (RPS) set to the 1-st state, the magnitude of the measurement, denote as $m_{n, r}$, can be expressed as

$$
m_{n, r}=\beta\left|\alpha_{1,1}+\alpha_{n, r} e^{j\left[\epsilon_{n, r}+\frac{\pi}{2}(r-1)\right]}\right|, 1 \leq r \leq 4,
$$

where $\beta$ is an unknown factor related to the non-linearity of amplifiers when two antenna elements are activated simultaneously. We model the phase errors being antenna-dependent, but consistent among different phase states, i.e. $\left\{\epsilon_{n, r}\right\}_{r=1}^{4}=\epsilon_{n}$. Then $\epsilon_{n}$ represents the phase error of the $n$-th antenna element. This model is reasonable since the phase error of the antennas are generally introduced by imperfect circuit designs and coarse PCB fabrications, and moreover all phase states of the same antenna suffer from the same hardware impairments, making the phase error depend on the antenna but be consistent among different phase states. Accordingly, the measurements in (18) can be rewritten as

$$
m_{n, r}=\beta\left|\alpha_{1,1}+\alpha_{n, r} e^{j\left[\epsilon_{n}+\frac{\pi}{2}(r-1)\right]}\right|, 1 \leq r \leq 4 .
$$

By choosing two arbitrary measurements of different phase states $r_{1}$ and $r_{2}$, we can eliminate the unknown factor $\beta$ by taking their ratio and then obtain

$$
\frac{m_{n, r_{1}}^{2}}{m_{n, r_{2}}^{2}}=\frac{\left|\alpha_{1,1}+\alpha_{n, r_{1}} e^{j\left[\epsilon_{n}+\frac{\pi}{2}\left(r_{1}-1\right)\right]}\right|^{2}}{\left|\alpha_{1,1}+\alpha_{n, r_{2}} e^{j\left[\epsilon_{n}+\frac{\pi}{2}\left(r_{2}-1\right)\right]}\right|^{2}},
$$

which can be further transformed into a solvable quadratic equation. Then, the phase error $\epsilon_{n}$ is found. To be more robust against inaccurate operations during data collections and the imperfectness of the model $\left\{\epsilon_{n, r}\right\}_{r=1}^{4}=\epsilon_{n}$, we vary $r_{1}$ and $r_{2}$ to get six different equations of the form in (20) and take the average over all the possibilities of $\epsilon_{n}$.

\subsection{Discussions on calibration result}

First, the received SNR with each antenna individually activated is shown in Fig. 4. As we can see, each antenna element provides a different antenna gain, even when a different phase state is applied. This observation confirms that it is necessary to determine the antenna gain for each element so that the desired radiation pattern can be generated accurately.

More importantly, we validated our calibration performance for directional beam patterns in terms of receiver SNR, which is shown in Fig. 5. The experimental validation of the phased array calibration is detailed as follows. Taking the Tx side as an example: firstly, the Tx and Rx phased arrays are mechanically aligned. Secondly, the Tx phased array is manually rotated towards a direction, i.e. a predefined AoD, while the Rx phased array is kept fixed for the whole validation process. Thirdly, all the Tx antenna elements are activated and the phase shifters are configured to generate a directional beam pointing to this AoD while only one Rx antenna is turned on for receiving the signal. Similarly, to validate the calibration at the Rx side, the antenna activation settings of these two phased arrays are switched so that the Tx transmits the signal with a single antenna turned on while all the $12 \mathrm{Rx}$ antennas are activated and configured to certain AoAs.

For both Tx and Rx sides, 19 directions, varying from $-45^{\circ}$ to $45^{\circ}$ in steps of $5^{\circ}$, are tested. As we can see from Fig. 5, an averaged SNR improvement of around $4 \mathrm{~dB}$ is achieved for both Tx and $\mathrm{Rx}$ sides, which indicates that much better link performance can be achieved by using the calibrated directional beams. Moreover, by comparing the receiver SNRs after calibration among different directional beams, we can observe that different directional beams result in a similar receiver SNR. This indicates that a similar antenna gain is provided by these calibrated directional beams, which further verifies our calibration process.

\section{EXPERIMENTAL VALIDATION}

In this section, we examine the proposed non-coherent beam alignment algorithm on our mmWave testbed. Note that the algorithm is developed based on ULAs. Our phased arrays, however, are not perfect ULAs since the antenna elements have non-isotropic gain patterns shown in Fig. 4 and the elevation beam width is $20^{\circ}$. Nevertheless, we can still use them to evaluate our algorithm for the following two motivations: (1) our phased array calibration helps to compensate the non-isotropic behaviors to some extends, which is shown in Fig. 5. (2) both the arrays are placed at the same height 


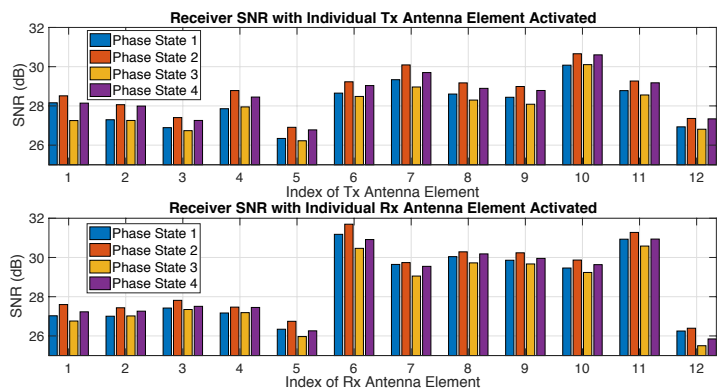

Figure 4: Element gain measurement

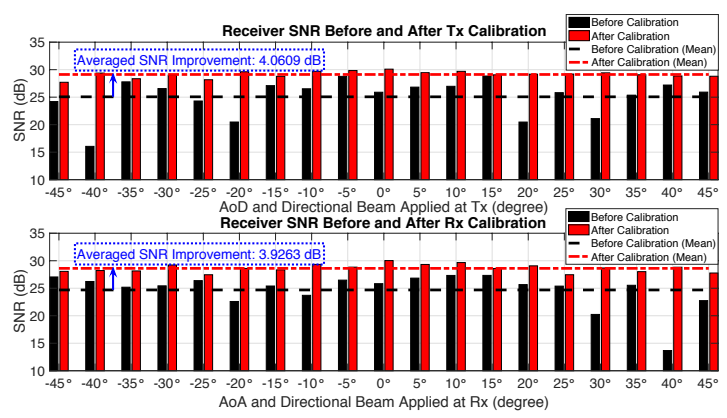

Figure 5: Performance improvement achieved by calibration

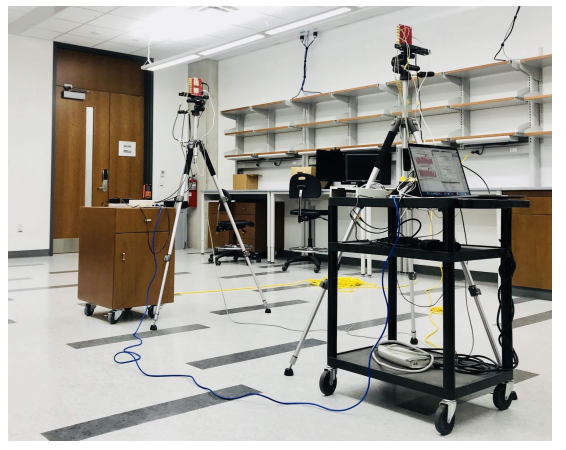

Figure 6: Experimental environment

during the experiments, making them work within the applicable vertical range.

Experimental environment and validation methodology: The experiments were performed at several different places which all provide a LOS path and do not have too much reflection like shown in Fig. 6. It is thus reasonable to assume that the ground truth of the channel can be modeled as (4). Accordingly, we can imitate a channel $\mathbf{H}=h_{1} \mathbf{a}_{\mathrm{Rx}}\left(\theta_{\text {Test }}\right) \mathbf{a}_{\mathrm{Tx}}^{*}\left(\phi_{\text {Test }}\right)$ by rotating the Tx phased array by $\phi_{\text {Test }}$ degrees and rotating Rx phased array by $\theta_{\text {Test }}$ degrees. Based on this imitated real channel, we validate our algorithm and compare it to the corresponding numerical results. We validated the proposed non-coherent beam alignment algorithm in terms of the mean angle estimation error (MAEE, see Appendix A for a mathematical definition) and receiver SNR. Two tests with different numbers of measurements were performed. In Test 1 , six training beams were used with the searching regions of AoD and AoA set as $\left[-22.5^{\circ}, 22.5^{\circ}\right]$. In Test 2 , eight training beams were used with the searching regions set as $\left[-25^{\circ}, 25^{\circ}\right]$. For both tests, the developed algorithm was examined under 18 different channel settings by either fixing $\theta_{\text {Test }}=0^{\circ}$ and varying $\phi_{\text {Test }}$ from $-20^{\circ}$ to $20^{\circ}$ in steps of $5^{\circ}$ or fixing $\phi_{\text {Test }}=0^{\circ}$ and varying $\theta_{\text {Test }}$ from $-20^{\circ}$ to $20^{\circ}$ in steps of $5^{\circ}$. In the experiments, the algorithm was first used to estimate the AoD and AoA of the LOS path and then the phased arrays were steered to the estimated angles by reconfiguring the phase shifters.

Result discussions: The MAEE performance is shown in Fig. 7a and Fig. 7c, two major observations can be concluded as follows: (1) as expected, the proposed non-coherent estimation algorithm outperforms the beam sweeping strategy by providing much smaller MAEE for the AoA/AoD of the dominant path. Besides, in some cases such as shown in the left sub-figure of Fig. 7a, the experimental results are close to the simulated ones. (2) It can be observed that, for the beam sweeping strategy, there are some unexpected beam misalignments when comparing the experimental results and the simulated ones. These misalignments could be due to several possibilities and one potential factor should be the residual errors from the calibration process. In particular, for Test 2 (shown in Fig. 7c), we can see that a more significant misalignment in beam sweeping strategy and a poorer MAEE performance of the noncoherent estimation happen concurrently. This not only shows the imperfectness of the performed calibration but also reveals that the calibration of phased arrays is of great importance, even for the simplest beamforming method.

In Fig. 7b and Fig. 7d, the performance of receiver SNR after beam alignment are further demonstrated. Overall, a better SNR is achieved by our proposed non-coherent beam alignment algorithm in comparison with the best SNR obtained by the beam sweeping strategy. This confirms the superiority of the proposed algorithm. Nevertheless, we can see that the SNR improvement becomes smaller when more measurements are employed. This is because a larger number of measurements will result in more overlapped beam patterns, which makes the beam sweeping strategy become closer to the optimal performance. Hence, the improvement achieved by the developed algorithm is relatively less significant.

\section{RELATED WORK}

Side information: Sharing position information will be an indispensable feature for future wireless systems, e.g. vehicular communications [9]. In [9, 11, 16], the idea of using the position information from GPS was initially proposed to reduce the beam pointing overhead of the LOS mmWave channels in vehicular communication settings. In [1], a multi-level beam search strategy was designed by considering location uncertainty. In [22], a compressive channel estimation and tracking strategy was developed by leveraging position and trajectory information of users. Both [1] and [22], however, assumed the perfect phase information of the measurements. In NLOS conditions, the data-driven approaches such as machine learning [33] and database [10,29] were further combined with position information to improve the beam prediction accuracy with little overheads. In addition to position information, many other sensor systems are ready to provide side information to help infer 


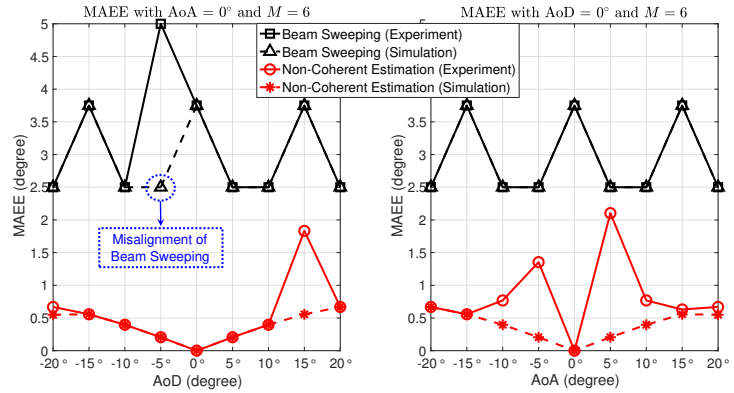

(a) $S_{\phi}=S_{\theta}=\left[-22.5^{\circ}, 22.5^{\circ}\right]$

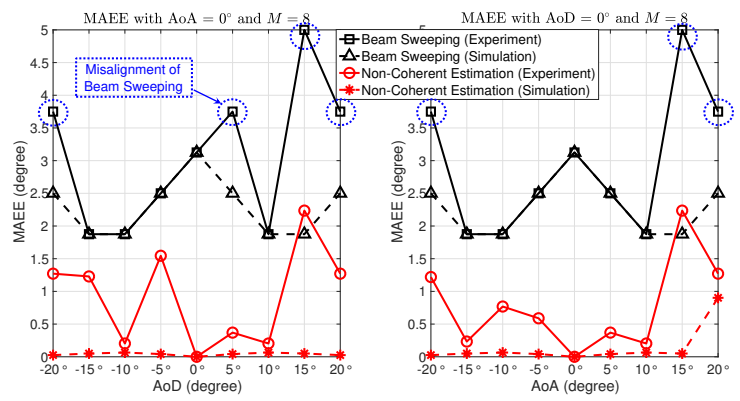

(c) $S_{\phi}=S_{\theta}=\left[-25^{\circ}, 25^{\circ}\right]$

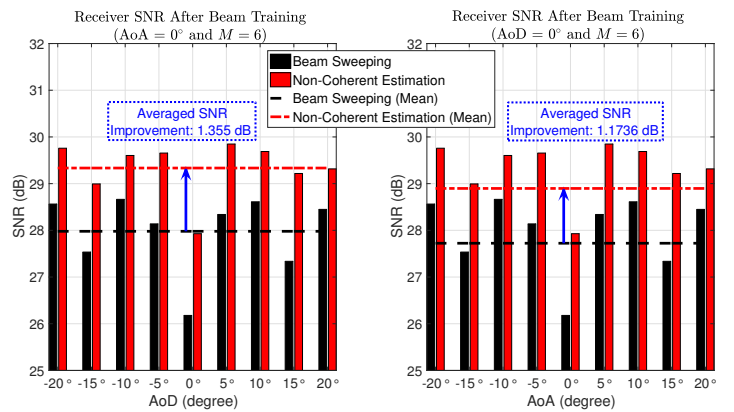

(b) $S_{\phi}=S_{\theta}=\left[-22.5^{\circ}, 22.5^{\circ}\right]$

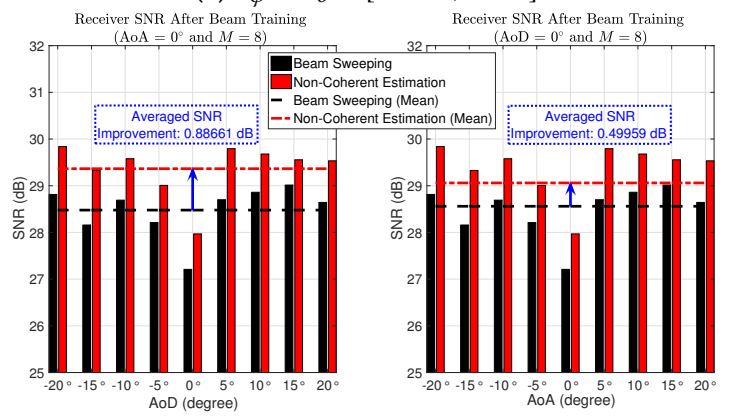

(d) $S_{\phi}=S_{\theta}=\left[-25^{\circ}, 25^{\circ}\right]$

Figure 7: Experimental validation of non-coherent estimation. Test 1: (a) and (b). Test 2: (c) and (d)

mmWave channel [12]. For example, the communication modules operating at the frequencies out of mmWave bands can be potentially leveraged. In [3] and [18], the dominant spatial directions of the mmWave channel were extracted from the sub- $6 \mathrm{GHz}$ signals to accelerate the beam training. The prior work showed that a significant overhead reduction is achievable by using side information, in comparison with the exhausted beam sweeping strategy given by IEEE 802.11ad [1, 3, 11, 18, 29].

MmWave prototypes: The mmWave testbed development has been initiated by the industry. In [23], one of the pioneering mmWave testbeds was demonstrated by Samsung Electronics Co., Ltd., in which a large number of measurements were taken to show the feasibility of mmWave with low-complexity analog beamforming algorithms. In academia, some researchers started by using mechanically steerable horn antennas to emulate the electronically-steerable phased arrays $[13,26]$. More recently, the electronically-steerable phased arrays have been exploited in mmWave prototyping. In [34] and [24], two $60 \mathrm{GHz}$ mmWave testbeds were developed with reconfigurable phased arrays but fixed beam codebooks. The OpenMili developed in [34] is based on the off-the-shelf FPGA processors and the X60 developed in [24] is based on NI Phased Arrays Toolkit. These two testbeds are constrained by their fixed codebooks, high price and limited flexibility for fast prototyping. In contrast to the existing prototypes, our developed mmWave testbed has benefited from our one-time calibration procedure, hence being able to generate precise directional beams.

Non-coherent algorithms: A few non-coherent algorithms have been proposed to tolerate the phase noise among measurements. In [14], a random hashing and voting mechanism was developed to perform the beam training in full angular space, hence resulting in a large training overhead. In [21], a non-coherent compressive path estimation was developed, in which the correlation between the nominal received signal strength (RSS) and the experimental RSS was used to determine the dominant channel path. This work focused on a single-sided beam training model and used pseudorandom training beacons, which however limits the communication range and degrades the SNR for the training phase as well. In [25], based on the compressive path estimation algorithm proposed in [21], a 3D beam training prototype with commodity IEEE 802.11ad router (Talon AD7200) was developed. However, all the employed beam patterns in [25] had to be carefully measured before being used to calculate the correlation, which greatly degrades the flexibility of its developed testbed. In a very recent work [19], an adaptive codebook optimization (ACO) method was proposed to optimize the beamforming patterns with multiple non-coherent measurements. However, the ACO method requires at least four probes per antenna for every training phase, which results in large overhead. Instead, our one-time calibration enables compressive channel estimation, hence avoiding training each phase state per antenna as in [19]. The CPR technique was studied in [20] for estimating the mmWave channel with non-coherent measurements. This work has similar limitations of [21] in adopting the omnidirectional reception mechanism and pseudo-random beams. Different from [20], our developed algorithm works for both single-sided and double-sided beam training frameworks and leverages side information as well as directional beams to improve the beam training performance, which has been furthermore justified by the real measurements. 


\section{CONCLUSIONS}

In this work, we have shown the feasibility of using the CPR technique to estimate the sparse mmWave channel with non-coherent measurements. The proposed two-stage CPR algorithm has exploited the properties of phase retrieval (PR) and the classic compressive sensing (CS). Alongside the theoretical formulation, we have developed a fully reconfigurable mmWave testbed by effectively calibrating low-cost custom-made phased arrays. The proposed non-coherent algorithm has been successfully implemented on the testbed and the consistency between the experimental results and theoretical analysis have well validated our proposed algorithm. We believe that our non-coherent algorithm and mmWave testbed can not only bring us closer to the more complicated mmWave algorithm designs but also provide more insights into the potential difficulties in realizing practical mmWave systems.

\section{ACKNOWLEDGMENTS}

This work was partially supported by the U.S. Army Research Labs under grant W911NF-19-1-0221, NSF under grants CNS-1343383, CNS-1731658, CNS-1702800, the Wireless Networking and Communications Group Industrial Affiliates Program, and the US DoT supported D-STOP Tier 1 University Transportation Center.

\section{A NUMERICAL SIMULATIONS}

In this Appendix, we numerically evaluate the average performance of the proposed non-coherent beam alignment algorithm. In the numerical simulation, both the Tx and Rx employ a ULA with $d=$ $3.055 \mathrm{~mm}$ and the carrier frequency is $60.48 \mathrm{GHz}$. Each numerical result is obtained by solving the estimation problem in (13) for 2000 times and then taking the average, with randomly generated channel realization following a Rician channel model given as

$$
\mathbf{H}=\sqrt{\frac{K}{K+1}} \underbrace{h_{1} \mathbf{a}_{\mathrm{Rx}}\left(\theta_{1}\right) \mathbf{a}_{\mathrm{Tx}}^{*}\left(\phi_{1}\right)}_{\text {Dominant path }}+\sqrt{\frac{1}{K+1}} \sum_{\ell=2}^{L} h_{\ell} \mathbf{a}_{\mathrm{Rx}}\left(\theta_{\ell}\right) \mathbf{a}_{\mathrm{Tx}}^{*}\left(\phi_{\ell}\right),
$$

where $L=5$ and the Rician K-factor is set to $7 \mathrm{~dB}$. The AoD and AoA of the dominant path is assumed to be uniformly distributed in the searching region $S_{\phi}$ and $S_{\theta}$, while the other $L-1$ paths are uniformly distributed in the full angular space. Without loss of generality, we set $S_{\phi}$ and $S_{\theta}$ to the same value and to be symmetric with respect to the broadside of ULA, i.e., $S_{\phi}=S_{\theta}=\left[-\frac{\Delta \theta}{2}, \frac{\Delta \theta}{2}\right]$. In addition, we let an equal number of beam patterns be applied at both Tx and Rx sides, which yields $M_{\mathrm{Tx}}=M_{\mathrm{Rx}} \triangleq M$ and thus the total number of measurements is $M^{2}$. The gird size of spatial angles are set to the same value, i.e. $G_{\mathrm{Tx}}=G_{\mathrm{Rx}} \triangleq G$. The regularized parameter $\eta$ is set to 0.05 and the threshold $\gamma$ used in the lowrank decomposition is set to 0.8 . Other required parameters will be specified in the corresponding simulation. To solve the PR problem in (14), we use the Templates for First-Order Conic Solvers (TFCOS) software package [6]. Regarding the CS problem in (13b), we choose the EMBGAMP [32] as our solver.

For comparison, we also provide the simulation results of three typical benchmarks: the first one is an ideal case where the coherent measurements with perfect phase information are available and EMBGAMP is directly applied to estimate the channel, which is labeled as Perfect Phase CS. The second one is a more practical

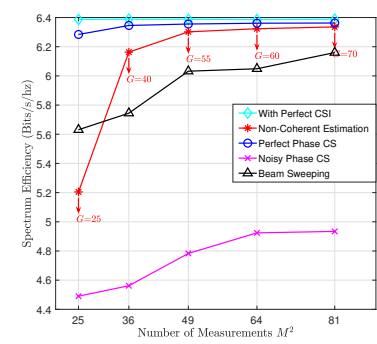

(a) SE vs. $M^{2}$

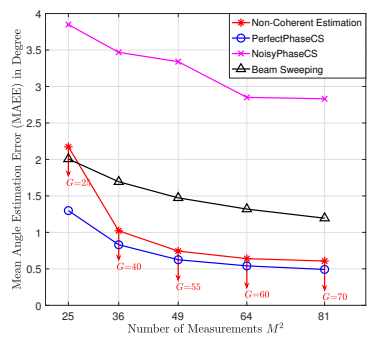

(b) MAEE vs. $M^{2}$
Figure 8: Numerical validation
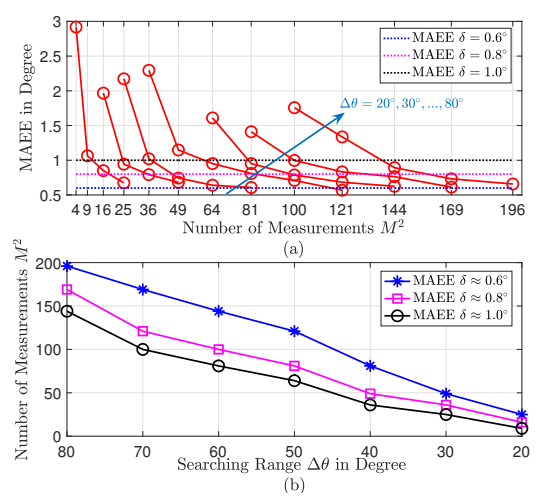

Figure 9: (a) MAEE, denoted as $\delta$, versus number of measurements $M^{2}$ for different searching range $\Delta \theta$. (b) An alternative presentation of data in (a) by approximately showing the tendency of $M^{2}$ versus $\Delta \theta$ for achieving different levels of MAEE $\delta$.

scenario where randomly generated phase noises are assigned to the simulated coherent measurements and then EMBGAMP is directly applied to estimate the channel, which is labeled as Noisy Phase CS. The third benchmark is the beam sweeping strategy that directional beam patterns are probed within the searching regions, which is labeled as Beam Sweeping. Our proposed algorithm is label as Non-Coherent Estimation. All the above algorithms use overlapped directional beams. This is because random beam patterns inevitably disregard the side information, hence degrading the SNR and boosting the measurement number.

The nature of the proposed non-coherent beam alignment algorithm is to estimate the AoD and AoA of the dominant path, and steer the analog transmit and receive beams towards the estimated angles. Thus, we evaluate the algorithm in terms of spectral efficiency (SE) and mean angle estimation error (MAEE) in comparison with the aforementioned benchmarks. Herein, the MAEE is defined as $\mathbb{E}\left[\left|\zeta_{\text {True }}-\zeta_{\text {Est }}\right|\right]$, where $\zeta_{\text {True }}$ represents the exact AoD or AoA of the dominant path in degree, while $\zeta_{\text {Est }}$ is its estimate.

The SE performance is shown in Fig. 8a and the MAEE performance is demonstrated in Fig. 8b. The searching regions of AoD and AoA are both set as $\left[-20^{\circ}, 20^{\circ}\right]$. The SNR before beam training is $0 \mathrm{~dB}$. First, from both Fig. $8 \mathrm{a}$ and Fig. $8 \mathrm{~b}$, we can see that given a 
reasonable number of measurements, the proposed non-coherent beam alignment achieves almost the same performance as the ideal scenario where perfect phase information is available. Second, it can be seen that the direct usage of the classic CS solver to the noncoherent measurements will result in a worse estimation performance compared with other strategies. This observation confirms the importance of using the non-coherent estimation method when the perfect phase information of measurements is not available.

In Fig. 9, we further show the benefits of side information in reducing the beam training overhead by evaluating the MAEE performance of our algorithm under the different searching range $\Delta \theta$. The SNR before beam training is set as $0 \mathrm{~dB}$. As we can see from Fig. 9 , the required number of measurements decreases significantly as the searching range $\Delta \theta$ shrinks. This is because the more accurate the side information is, the fewer is the number of directional beams required to find the angles of the dominant path.

\section{REFERENCES}

[1] Ahmed Abdelreheem, Ehab Mahmoud Mohamed, and Hamada Esmaiel. 2018 Location-Based Millimeter Wave Multi-Level Beamforming Using Compressive Sensing. IEEE Communications Letters 22, 1 (Jan. 2018), 185-188.

[2] Mehmet Akçakaya and Vahid Tarokh. 2015. Sparse Signal Recovery from a Mixture of Linear and Magnitude-Only Measurements. IEEE Signal Processing Letters 22, 9 (Sept. 2015), 1220-1223.

[3] Anum Ali, Nuria González-Prelcic, and Robert W. Heath. 2018. Millimeter Wave Beam-Selection Using Out-of-Band Spatial Information. IEEE Transactions on Wireless Communications 17, 2 (Feb. 2018), 1038-1052.

[4] Ahmed Alkhateeb, Omar El Ayach, Geert Leus, and Robert W. Heath. 2014 Channel Estimation and Hybrid Precoding for Millimeter Wave Cellular Systems. IEEE Journal of Selected Topics in Signal Processing 8, 5 (Oct. 2014), 831-846.

[5] Sohail Bahmani and Justin Romberg. 2015. Efficient Compressive Phase Retrieval with Constrained Sensing Vectors. In Proc. of the 28th International Conference on Neural Information Processing Systems - Volume 1 (NIPS’15). MIT Press, 523-531.

[6] Stephen R. Becker, Emmanuel J. Candès, and Michael C. Grant. 2011. Templates for Convex Cone Problems with Applications to Sparse Signal Recovery. Mathematical Programming Computation 3, 3 (July 2011), 165-218.

[7] Emmanuel J. Candès, Yonina C. Eldar, Thomas Strohmer, and Vladislav Voroninski. 2013. Phase Retrieval via Matrix Completion. SIAM fournal on Imaging Sciences 6, 1 (2013), 199-225.

[8] Emmanuel J. Candès, Thomas Strohmer, and Vladislav Voroninski. 2013 PhaseLift: Exact and Stable Signal Recovery from Magnitude Measurements via Convex Programming. Communications on Pure and Applied Mathematics 66, 8 (2013), 1241-1274.

[9] Junil Choi, Vutha Va, Nuria González-Prelcic, Robert Daniels, Chandra R. Bhat, and Robert W. Heath. 2016. Millimeter-Wave Vehicular Communication to Support Massive Automotive Sensing. IEEE Communications Magazine 54, 12 (Dec. 2016), 160-167.

[10] Ilario Filippini, Vincenzo Sciancalepore, Francesco Devoti, and Antonio Capone. 2018. Fast Cell Discovery in Mm-Wave 5G Networks With Context Information. IEEE Transactions on Mobile Computing 17, 7 (July 2018), 1538-1552.

[11] Nil Garcia, Henk Wymeersch, Erik G. Ström, and Dirk Slock. 2016. Location-Aided Mm-Wave Channel Estimation for Vehicular Communication. In 2016 IEEE 17th International Workshop on Signal Processing Advances in Wireless Communications $(S P A W C)$. IEEE.

[12] Nuria González-Prelcic, Anum Ali, Vutha Va, and Robert W. Heath. 2017. Millimeter-Wave Communication with Out-of-Band Information. IEEE Communications Magazine 55, 12 (Dec. 2017), 140-146.

[13] Muhammad Kumail Haider and Edward W. Knightly. 2016. Mobility Resilience and Overhead Constrained Adaptation in Directional $60 \mathrm{GHz}$ WLANs: Protocol Design and System Implementation. In Proc. of the 17th ACM International Symposium on Mobile Ad Hoc Networking and Computing (MobiHoc '16). ACM, 61-70.

[14] Haitham Hassanieh, Omid Abari, Michael Rodriguez, Mohammed Abdelghany, Dina Katabi, and Piotr Indyk. 2018. Fast Millimeter Wave Beam Alignment. In Proc. of the 2018 Conference of the ACM Special Interest Group on Data Communication (SIGCOMM '18). ACM, 432-445.

[15] Mark Iwen, Aditya Viswanathan, and Yang Wang. 2017. Robust Sparse Phase Retrieval Made Easy. Applied and Computational Harmonic Analysis 42, 1 (Jan. 2017), 135-142.

[16] Flavio Maschietti, David Gesbert, Paul de Kerret, and Henk Wymeersch. 2017 Robust Location-Aided Beam Alignment in Millimeter Wave Massive MIMO. In
Proc. of 2017 IEEE Global Communications Conference (GLOBECOM 2017). IEEE.

[17] Thomas Nitsche, Carlos Cordeiro, Adriana B. Flores, Edward W. Knightly, Eldad Perahia, and Joerg C. Widmer. 2014. IEEE 802.11ad: Directional $60 \mathrm{GHz}$ Communication for Multi-Gigabit-per-second Wi-Fi. IEEE Communications Magazine 52, 12 (Dec. 2014), 132-141

[18] Thomas Nitsche, Adriana B. Flores, Edward W. Knightly, and Joerg Widmer. 2015. Steering With Eyes Closed: Mm-Wave Beam Steering Without In-Band Measurement. In 2015 IEEE Conference on Computer Communications (INFOCOM). IEEE, 2416-2424.

[19] Joan Palacios, Daniel Steinmetzer, Adrian Loch, Matthias Hollick, and Joerg Widmer. 2018. Adaptive Codebook Optimization for Beam Training on Off-theShelf IEEE 802.11Ad Devices. In Proc. of the 24th Annual International Conference on Mobile Computing and Networking (MobiCom '18). ACM, 241-255.

[20] Maryam Eslami Rasekh and Upamanyu Madhow. 2018. Noncoherent Compressive Channel Estimation for Mm-Wave Massive MIMO. (2018). arXiv:arXiv: 1801.06608

[21] Maryam Eslami Rasekh, Zhinus Marzi, Yanzi Zhu, Upamanyu Madhow, and Haitao Zheng. 2017. Noncoherent mmWave Path Tracking. In Proc. of the 18th International Workshop on Mobile Computing Systems and Applications (HotMobile '17). ACM, 13-18.

[22] Javier Rodriguez-Fernandez, Nuria González-Prelcic, and Robert W. Heath. 2018. Position-Aided Compressive Channel Estimation and Tracking for Millimeter Wave Multi-User MIMO Air-to-Air Communications. In 2018 IEEE International Conference on Communications Workshops (ICC Workshops). IEEE.

[23] Wonil Roh, Ji-Yun Seol, Jeongho Park, Byunghwan Lee, Jaekon Lee, Yungsoo Kim, Jaeweon Cho, Kyungwhoon Cheun, and Farshid Aryanfar. 2014. MillimeterWave Beamforming as an Enabling Technology for 5G Cellular Communications: Theoretical Feasibility and Prototype Results. IEEE Communications Magazine 52, 2 (Feb. 2014), 106-113.

[24] Swetank Kumar Saha, Yasaman Ghasempour, Muhammad Kumail Haider, Tariq Siddiqui, Paulo De Melo, Neerad Somanchi, Luke Zakrajsek, Arjun Singh, Owen Torres, Daniel Uvaydov, Josep Miquel Jornet, Edward Knightly, Dimitrios Koutsonikolas, Dimitris Pados, and Zhi Sun. 2017. X60: A Programmable Testbed for Wideband $60 \mathrm{GHz}$ WLANs with Phased Arrays. In Proc. of the 11th Workshop on Wireless Network Testbeds, Experimental Evaluation \& CHaracterization (WiNTECH '17). ACM, 75-82.

[25] Daniel Steinmetzer, Daniel Wegemer, Matthias Schulz, Joerg Widmer, and Matthias Hollick. 2017. Compressive Millimeter-Wave Sector Selection in Offthe-Shelf IEEE 802.11 ad Devices. In Proc. of the 13th International Conference on Emerging Networking EXperiments and Technologies (CoNEXT '17). ACM, 414425

[26] Sanjib Sur, Vignesh Venkateswaran, Xinyu Zhang, and Parmesh Ramanathan. 2015. $60 \mathrm{GHz}$ Indoor Networking Through Flexible Beams: A Link-Level Profiling. In Proc. of the 2015 ACM SIGMETRICS International Conference on Measurement and Modeling of Computer Systems (SIGMETRICS '15). ACM, 71-84.

[27] Tesla, Inc., CA, USA. 2018. Full self-driving hardware on all cars. Retrieved Nov. 13, 2018 from https://www.tesla.com/autopilot

[28] Joel A. Tropp and Anna C. Gilbert. 2007. Signal Recovery From Random Measurements Via Orthogonal Matching Pursuit. IEEE Transactions on Information Theory 53, 12 (Dec. 2007), 4655-4666.

[29] Vutha Va, Junil Choi, Takayuki Shimizu, Gaurav Bansal, and Robert W. Heath. 2018. Inverse Multipath Fingerprinting for Millimeter Wave V2i Beam Alignment. IEEE Transactions on Vehicular Technology 67, 5 (May 2018), 4042-4058.

[30] Alberto Valdes-Garcia, Bodhisatwa Sadhu, Xiaoxiong Gu, Yahya Tousi, Duixian Liu, Scott K. Reynolds, Joakim Haillin, Stefan Sahl, and Leonard Rexberg. 2018. Circuit and Antenna-in-Package Innovations for Scaled mmWave 5G Phased Array Modules. In 2018 IEEE Custom Integrated Circuits Conference (CICC). IEEE.

[31] Kiran Venugopal, Ahmed Alkhateeb, Nuria González-Prelcic, and Robert W. Heath. 2017. Channel Estimation for Hybrid Architecture-Based Wideband Millimeter Wave Systems. IEEE fournal on Selected Areas in Communications 35, 9 (Sept. 2017), 1996-2009.

[32] Jeremy Vila and Philip Schniter. 2011. Expectation-Maximization BernoulliGaussian Approximate Message Passing. In 2011 Conference Record of the Forty Fifth Asilomar Conference on Signals, Systems and Computers (ASILOMAR). IEEE, 799-803.

[33] Yuyang Wang, Murali Narasimha, and Robert W. Heath. 2018. MmWave Beam Prediction with Situational Awareness: A Machine Learning Approach. In 2018 IEEE 19th International Workshop on Signal Processing Advances in Wireless Communications (SPAWC). IEEE.

[34] Jialiang Zhang, Xinyu Zhang, Pushkar Kulkarni, and Parameswaran Ramanathan. 2016. OpenMili: A 60 GHz Software Radio Platform with a Reconfigurable Phasedarray Antenna. In Proc. of the 22nd Annual International Conference on Mobile Computing and Networking (MobiCom '16). ACM, 162-175.

[35] Yi Zhang, Kartik Patel, Sanjay Shakkottai, and Robert W. Heath. 2019. Open Source Code for Side-information-Aided Non-coherent Beam Alignment (SANBA) design and prototyping for MmWave Systems. Retrieved Apr. 8, 2019 from https://github.com/yzhang417/SANBA-mmWave-SDR 